\title{
Prediction of S-1 adjuvant chemotherapy benefit in Stage II/III gastric cancer treatment based on comprehensive gene expression analysis
}

\author{
Kenichi Nakamura ${ }^{1}$ Keiichi Hatakeyama ${ }^{2}$ Kenichiro Furukawa ${ }^{1} \cdot$ Keiichi Fujiya ${ }^{1} \cdot$ Satoshi Kamiya ${ }^{1}$. \\ Makoto Hikage ${ }^{1} \cdot$ Yutaka Tanizawa $^{1} \cdot$ Etsuro Bando $^{1} \cdot$ Keiichi Ohshima $^{2} \cdot$ Kenichi Urakami $^{3} \cdot$ Nozomu Machida $^{4}$. \\ Hirofumi Yasui ${ }^{4} \cdot$ Ken Yamaguchi ${ }^{5} \cdot$ Masanori Terashima $^{1}[$
}

Received: 7 December 2019 / Accepted: 29 February 2020 / Published online: 18 March 2020

(c) The International Gastric Cancer Association and The Japanese Gastric Cancer Association 2020

\begin{abstract}
Background Studies to identify predictive biomarkers of adjuvant chemotherapy with S-1 after gastrectomy in Stage II/III gastric cancer patients have been done; however, more clarity and understanding are needed. Our aim in the present study was to identify biomarkers predicting benefit due to $S-1$ adjuvant chemotherapy using comprehensive gene expression analysis. Methods We retrospectively analyzed 102 patients receiving adjuvant chemotherapy with S-1 and 46 patients not receiving S-1 adjuvant chemotherapy after gastrectomy for gastric cancer treatment between January 2014 and December 2016. Hierarchical clustering analysis was performed based on the gene expression data obtained using cDNA microarray. Differentially expressed genes (DEGs) were identified using thresholds of absolute fold changes of $>4.0$ and a false discovery rate $P$ value of $<0.01$. Gene Ontology (GO) analysis and GO network visualization were performed using the ClueGO app in Cytoscape. Results Hierarchical clustering analysis in patients treated with S-1 adjuvant chemotherapy revealed two clusters with favorable and unfavorable survival outcomes. We identified 147 upregulated DEGs and 192 downregulated DEGs in the favorable outcome group. GO analysis to identify significantly upregulated genes showed enrichment in immune-related genes and GO terms. Upregulation of these immune-related genes was not associated with survival in patients not receiving S-1 adjuvant chemotherapy.

Conclusions The upregulation and enrichment of immune-related genes and GO terms may be predictive biomarkers in patients who would benefit from adjuvant S-1 chemotherapy to treat Stage II/III gastric cancer.
\end{abstract}

Keywords Adjuvant drug therapy · Gastric cancer $\cdot$ Drug response biomarker $\cdot$ Gene expression profiling

\section{Introduction}

Electronic supplementary material The online version of this article (https://doi.org/10.1007/s10120-020-01056-6) contains supplementary material, which is available to authorized users.

Masanori Terashima

m.terashima@scchr.jp

1 Division of Gastric Surgery, Shizuoka Cancer Center, 1007 Shimonagakubo, Nagaizumi-cho, Sunto-gun, Shizuoka 411-8777, Japan

2 Medical Genetics Division, Research Institute, Shizuoka Cancer Center, 1007 Shimonagakubo, Nagaizumi-cho, Sunto-gun, Shizuoka 411-8777, Japan

3 Cancer Diagnostics Research Division, Research Institute, Shizuoka Cancer Center, 1007 Shimonagakubo, Nagaizumi-cho, Sunto-gun, Shizuoka 411-8777, Japan
Gastric cancer is the fifth most common cancer and the third leading cause of cancer death globally [1]. The main treatment strategy in gastric cancer is complete surgical

4 Division of Gastrointestinal Oncology, Shizuoka Cancer Center, 1007 Shimonagakubo, Nagaizumi-cho, Sunto-gun, Shizuoka 411-8777, Japan

5 Research Institute, Shizuoka Cancer Center, 1007 Shimonagakubo, Nagaizumi-cho, Sunto-gun, Shizuoka 411-8777, Japan 
resection with appropriate lymph node dissection. In Japan, adjuvant chemotherapy with S-1 (TS-1: Taiho Pharmaceutical, Tokyo, Japan) is recognized as the standard treatment for Stage II and III gastric cancer after curative resection [2] based on the findings of the Adjuvant Chemotherapy Trial of TS-1 for Gastric Cancer (ACTS-GC trial) [3]. This trial showed a $10 \%$ better five-year overall survival (OS) in the $\mathrm{S}-1$ adjuvant therapy group than in the surgery alone group. However, subset analysis revealed a five-year OS rate of only $50.2 \%$ in stage IIIB patients even in the S-1 group, suggesting a need for survival improvement [4]. Therefore, a better understanding of predictive biomarkers of S-1 benefit can help select patients for more effective treatment and improved survival outcomes.

A number of studies have reported that the expression of several enzymes involved in the S-1 metabolism pathway correlated with the clinical efficacy of adjuvant S-1 treatment. As a representative study, an exploratory biomarker analysis of ACTS-GC reported that high expression of thymidylate synthase (TS) and dihydropyrimidine dehydrogenase (DPD) was associated with enhanced benefit due to adjuvant S-1 treatment [5]. In contrast, other studies have shown that high expression of TS and DPD had a negative impact on the efficacy of S-1 [6, 7]. Furthermore, the relationship between S-1 efficacy and other S-1 metabolic enzymes including thymidine phosphorylase (TP) and orotate phosphoribosyltransferase (OPRT) has also been unclear [8-10]. It remains challenging to predict S-1 efficacy based on the expression of S-1 metabolism enzymes; novel predictive biomarkers are required.

The Cancer Genome Atlas (TCGA) describes the molecular subtypes of gastric cancer based on comprehensive genomic analysis using bioinformatic approaches to explore novel therapies in defined sets of patients [11]. As a similar attempt, the Shizuoka Cancer Center initiated the HOPE (High-tech Omics-based Patient Evaluation) project in 2014 to evaluate the molecular biology of cancer and identify new biomarkers and molecularly targeted agents [12]. In the present study, we aimed to identify the predictive biomarkers of S-1 adjuvant chemotherapy benefit using gene expression profiling (GEP).

\section{Materials and methods}

\section{Patients}

A total of 297 consecutive patients who underwent gastrectomy for gastric cancer treatment at the Division of Gastric Surgery of Shizuoka Cancer Center (Shizuoka, Japan) between January 2014 and December 2016 were enrolled in the project HOPE [12]. A total of 135 patients were excluded because they did not meet the criteria for adjuvant chemotherapy based on the ACTS-GC trial except for age [2, 3]. Thus, 162 patients with indications for adjuvant chemotherapy with S-1 were enrolled. Among these 162 patients, 116 patients initiated adjuvant chemotherapy with $\mathrm{S}-1$, and 14 patients discontinued, therefore, 102 patients received adjuvant S-1 (S-1 group) for one year (Supplementary Fig. 1). The remaining 46 patients (non-S-1 group) did not receive adjuvant chemotherapy for reasons including age, comorbidities and/or patient preference. We first performed gene expression analysis in the S-1 group to detect predictive biomarkers for the effect of adjuvant S-1 treatment. Next, we evaluated these results in the non-S-1 group to determine whether these biomarkers were predictive or prognostic factors. Tumor stage and histological type were determined per the Japanese Classification of Gastric Carcinoma 2nd English edition [2].

The research plan was designed as per the revised Ethical Guidelines for Human Genome/Gene Analysis Research in Japan (https://www.lifescience.mext.go.jp/files/pdf/n1115 _01.pdf) and the study protocol was approved by the Institutional Review Board of Shizuoka Cancer Center (Approval number \#25-33). Written informed consent was obtained from all participants.

\section{Clinical samples}

Clinical samples weighing $\geq 0.1 \mathrm{~g}$ were dissected from the tumor and the surrounding normal tissues in the surgical specimens. The tumor lesion was visually assessed by experienced pathologists as containing $\geq 50 \%$ tumor content. For RNA analysis, clinical samples were immersed in RNAlater solution (Thermo Fisher Scientific, Waltham, MA, USA), minced and stored at $4{ }^{\circ} \mathrm{C}$ before RNA extraction. Total RNA was isolated from tissue samples using a miRNeasy Mini Kit (QIAGEN) following the manufacturer's recommendations. The extracted RNA was quantified using NanoDrop and the Qubit 2.0 Fluorometer (Thermo Fisher Scientific). We used RNA samples with RNA integrity number $\geq 6.0$ for gene expression analysis [13].

\section{CDNA microarray}

Purified RNA was amplified and fluorescently labelled using the One-color Low Input Quick Amp Labeling kit (Agilent Technologies) following the manufacturer's protocol. Gene expression analysis was performed on a SurePrint G3 Human Gene Expression $8 \times 60$ K v2 Microarray (Agilent Technologies), which has 50,599 probes. The raw signal intensity derived from the scanned image was filtered using Agilent Flag Values to maintain the reliability of the microarray data, and then log-transformed and normalized to the 75th percentile using the software GeneSpring (Agilent Technologies). The fold change in the expression between 
tumor tissues and normal mucosa in each patient was calculated using the $Z$ scores of the target genes [14, 15]. Microarray analysis was performed per MIAME guidelines [16].

\section{Gene expression analysis}

To reveal distinguishable groups among the patients, an unsupervised hierarchical clustering analysis using Ward's minimum variance method was performed based on the expression levels of all probe targets and the results were visualized as a heat map. Differentially expressed genes (DEGs) were identified with a fold change (FCs) of $>+4.0$ or $<-4.0$ and an FDR; Benjamini-Hochberg method) $P$ value of $<0.01$ was determined as cut-off criteria. GO analysis and GO network visualization were performed using the plug-in ClueGO [17] app in the software Cytoscape (v3.7.1) to identify over-represented GO categories in the biological processes. The GO terms were considered as being significantly enriched at a threshold of FDR $P$ value $<0.01$. With a Kappa score of 0.40 as a cut-off criterion, GO terms involved in more than five genes were selected to build the GO network. The minimum GO tree interval level was 2 and the maximum level was 6 , which was considered to represent low-to-medium network specificity. We further constructed the protein-protein interaction (PPI) network of the DEGs using the plug-in STRING app in Cytoscape. The confidence score cut-off was $>0.4$. We identified interactive genes using the degree method with a threshold of two interactions.

\section{Statistical analysis}

OS was defined as the time from surgery to death from any cause. Relapse-free survival (RFS) was defined as the time from surgery to relapse or death from any cause. The Chisquared test was performed for categorical data and the Wilcoxon rank-sum test was performed for continuous data. FDR $P$ value was used for multiple comparisons. For survival analysis, the Kaplan-Meier method was used to assess the survival time distribution and the log-rank test was used for comparisons. Statistical differences were considered significant at $P<0.05$ and FDR $P<0.01$. All statistical calculations were performed using the software JMP version 11.2.1 (SAS Institute Inc., Cary, NC, USA).

\section{Results}

\section{Patient characteristics}

Table 1 shows clinical and pathological characteristics of 102 patients who underwent adjuvant chemotherapy with S-1 (S-1 group) and 46 patients who did not receive adjuvant S-1 (non-S-1 group). There were no significant differences between the two groups, except for age. The median followup period was 37.1 months. 13 and 24 patients in S-1 group and non-S-1 group died during the follow-up period. The number of the gastric cancer-related death was 12 of 13 (92\%) in S-1 group, while 17 of 24 (71\%) in non-S-1 group $(P=0.11)$.

\section{Hierarchical clustering analysis}

Using an unsupervised hierarchical clustering analysis based on all probes, patients who underwent adjuvant S-1 therapy were divided into two clusters with different recurrence rates (Fig. 1a); all of 28 patients in cluster A had no recurrences of gastric cancer, whereas 21 out of 74 (28.4\%) patients in cluster $\mathrm{B}$ had recurrences $(P=0.005)$. Consequently, cluster A exhibited a better survival outcome than cluster B $(P=0.019$ in OS and $P=0.002$ in RFS; Fig. 1b, c). No significant differences were observed between the two clusters in terms of clinical or pathological characteristics except for tumor location (Table 2).

\section{Expression of key enzymes in the S-1 metabolism pathway}

We compared the gene expression of four key enzymes in the S-1 metabolism pathway, namely $D P D, T S, T P$ and $O P R T$, between the two clusters (Fig. 1d, Table 2). The expression of $T S$ was significantly higher in cluster A than in cluster $\mathrm{B}(P=0.0003)$. In contrast, the expression of $T P$ was significantly lower in cluster A than in cluster B $(P<0.0001)$. There were no significant differences in the expression of $D P D(P=0.074)$ or $O P R T(P=1.000)$ between the two clusters.

\section{Identification of DEGs and GO analysis}

A total of 339 DEGs were identified using the thresholds of absolute FC $>4.0[=|\log 2(\mathrm{FC})|>2.0]$ and FDR $P$ value $<0.01[=-\log 10($ FDR $P$ value $>1.8)]$. A volcano plot was used to visualize these DEGs between clusters A and B (Fig. 2a). In cluster A, 147 genes were upregulated and 192 genes were downregulated in comparison with cluster B. All the DEGs are listed in Supplementary Table S1. None of the genes $T S, D P D, T P$, or $O P R T$ in the S-1 metabolism pathway were found among these DEGs.

GO analysis was performed on the 147 upregulated DEGs and the 192 downregulated DEGs in cluster A. The GO term analysis for upregulated DEGs in cluster A identified 67 significant GO terms which were classified into 14 clusters. Immune-related GO terms comprised a large proportion of these GO terms; 10 out of the 14 clusters included immunerelated GO terms. The top three significant GO clusters are listed in Fig. 2b; all significant GO terms are listed in 


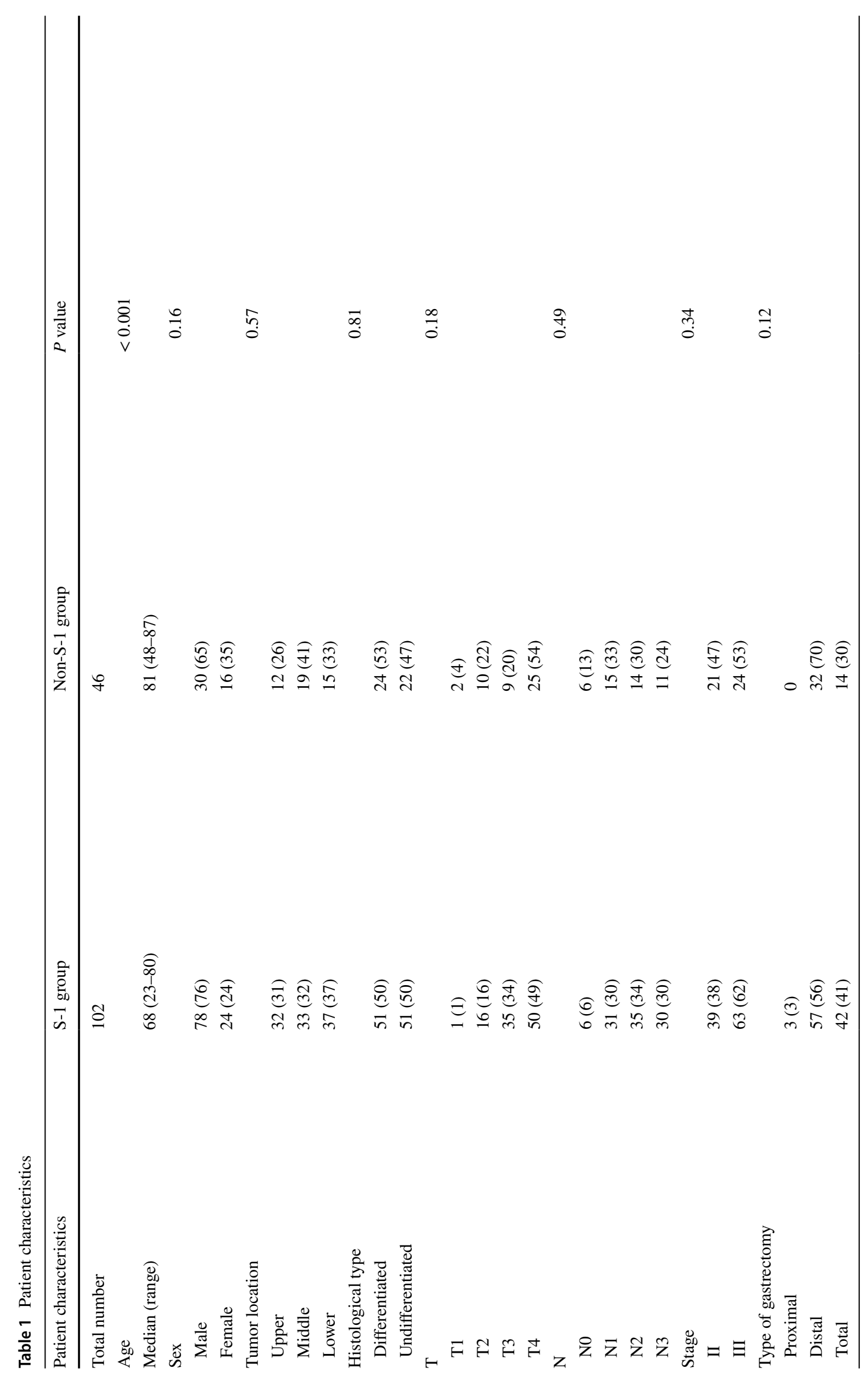



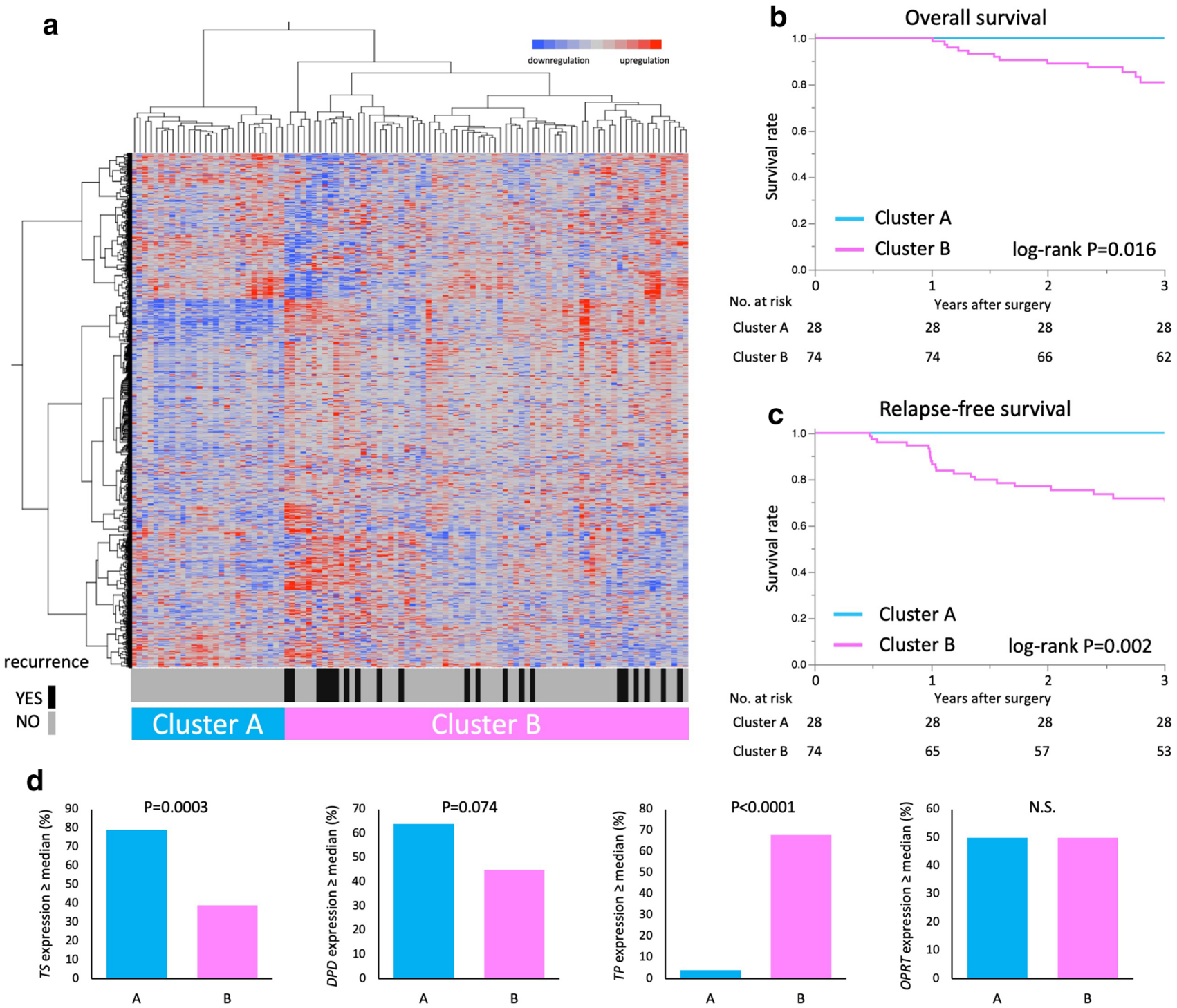

Fig. 1 Hierarchical clustering analysis. a Heatmap based on hierarchical clustering analysis. The color range from blue to red represents the range of gene expression from a downregulated state to an upregulated state. The horizontal axis represents patients and the vertical

Supplementary Table S2. The GO network is visualized in Fig. 2c. No significant GO terms were described in the GO analysis of downregulated DEGs in cluster A.

\section{PPI network analysis}

We analyzed the PPI network of the 147 upregulated DEGs in cluster A. Figure 3 shows a PPI network of 52 genes with more than two degrees of interaction. Several chemokines (CCL18, CCL19, CCL20, CCL21, CXCL13, CCR7, CCR9 and $C X C R 5$ ) were identified as strongly interacting genes.

axis represents gene clusters. b OS curve of patients in clusters A and B. c RFS curve of patients in clusters A and B. d Proportion of high S-1 metabolic enzyme (TS, DPD, TP and $O P R T)$ expression in clusters $\mathrm{A}$ and $\mathrm{B}$

\section{Prognostic impact of immune-related genes}

We verified whether upregulation of these immune-related genes would impact survival outcomes even in the nonS-1 group. We performed hierarchical clustering using 36 immune-related genes out of the 147 upregulated DEGs in cluster A. Patients were divided into cluster $\mathrm{X}$ with overexpression of these genes and cluster $\mathrm{Y}$ with under- or intermediate expression ( 21 in cluster $\mathrm{X}$ and 81 in cluster $\mathrm{Y}$ in the $\mathrm{S}-1$ group, and eight in cluster $\mathrm{X}$ and 38 in cluster $\mathrm{Y}$ in the non-S-1 group; Fig. 4a). Table 3 shows the patient characteristics of cluster $\mathrm{X}$ and $\mathrm{Y}$ in each group. There were no significant differences between the two groups. As 
Table 2 Comparison of patient characteristics between clusters A and B

\begin{tabular}{lll}
\hline Patient characteristics & Cluster & $P$ value \\
\cline { 2 - 3 } & $\mathrm{A}(n=28)$ & $\mathrm{B}(n=74)$
\end{tabular}

\begin{tabular}{|c|c|c|c|}
\hline Age & & & 0.321 \\
\hline Median (range) & $67(23-77)$ & $69(31-80)$ & \\
\hline Sex & & & 0.757 \\
\hline Male & $22(79)$ & $56(76)$ & \\
\hline Female & $6(21)$ & $18(24)$ & \\
\hline Tumor location & & & 0.023 \\
\hline Upper & $9(32)$ & $23(31)$ & \\
\hline Middle & $4(14)$ & $29(39)$ & \\
\hline Lower & $15(54)$ & $22(30)$ & \\
\hline Histological type & & & 0.657 \\
\hline Differentiated & $13(46)$ & $38(51)$ & \\
\hline Undifferentiated & $15(54)$ & $36(49)$ & \\
\hline $\mathrm{T}$ & & & 0.798 \\
\hline $\mathrm{T} 1$ & 0 & $1(1)$ & \\
\hline $\mathrm{T} 2$ & $4(14)$ & $12(16)$ & \\
\hline $\mathrm{T} 3$ & $11(39)$ & $24(33)$ & \\
\hline $\mathrm{T} 4$ & $13(47)$ & $37(50)$ & \\
\hline $\mathrm{N}$ & & & 0.3140 \\
\hline No & $1(4)$ & $5(7)$ & \\
\hline N1 & $11(39)$ & $20(27)$ & \\
\hline $\mathrm{N} 2$ & $11(39)$ & $24(33)$ & \\
\hline N3 & $5(18)$ & $25(33)$ & \\
\hline Stage & & & 0.433 \\
\hline II & $9(32)$ & $30(41)$ & \\
\hline III & $19(68)$ & $44(59)$ & \\
\hline Type of gastrectomy & & & 0.059 \\
\hline Proximal & $2(7)$ & $1(1)$ & \\
\hline Distal & $19(68)$ & $38(51)$ & \\
\hline Total & $7(25)$ & $35(48)$ & \\
\hline TS expression & & & 0.0003 \\
\hline$\geq$ median & $22(79)$ & $29(39)$ & \\
\hline$<$ median & $6(21)$ & $45(61)$ & \\
\hline DPD expression & & & 0.074 \\
\hline$\geq$ median & $18(64)$ & $33(45)$ & \\
\hline$<$ median & $10(36)$ & $41(55)$ & \\
\hline TP expression & & & $<0.0001$ \\
\hline$\geq$ median & $1(4)$ & $50(68)$ & \\
\hline$<$ median & $27(96)$ & $34(32)$ & \\
\hline OPRT expression & & & 1 \\
\hline$\geq$ median & $14(50)$ & $37(50)$ & \\
\hline$<$ median & $14(50)$ & $37(50)$ & \\
\hline
\end{tabular}

Statistically significant $P$ values are in bold $(P<0.05)$

$T S$ thymidylate synthase, $D P D$ dihydropyrimidine dehydrogenase, $T P$ thymidine phosphorylase, $O P R T$ orotate phosphoribosyltransferase expected, patients receiving adjuvant S-1 chemotherapy in cluster $\mathrm{X}$ exhibited better survival outcomes than those in cluster Y (Fig. 4b). However, there was no significant difference in the survival outcome between clusters $\mathrm{X}$ and $\mathrm{Y}$ in patients who did not receive adjuvant $\mathrm{S}-1$ chemotherapy (Fig. 4c).

Furthermore, we analyzed the age-based expression of these immune-related genes in the S-1 and the non-S-1 groups and found no significant changes in either group (Supplementary Fig. 2a, b).

\section{Discussion}

In the present study, we carried out gene expression and GO analyses to find predictive biomarkers to identify patients who may benefit from adjuvant S-1 chemotherapy. Hierarchical clustering analysis identified two groups with favorable (cluster A) or unfavorable (cluster B) survival outcomes. GO analysis for significantly upregulated genes in the favorable outcome group showed that immune-related genes and GO terms were enriched. Upregulation of these immune-related genes was not associated with survival in the patients not receiving adjuvant S-1 chemotherapy. This suggested that upregulation of immune-related genes might be a predictive biomarker of adjuvant S-1 chemotherapy benefit, but it may not be prognostic.

Many studies have reported the relationship between survival outcome and expression of S-1 metabolism enzymes [5-10]. The ACTS-GC biomarker exploratory study demonstrated that high expression of TS and DPD improved survival benefit due to adjuvant S-1 therapy [5]. In contrast, previous studies have reported that high expression of TS and DPD had a negative impact on the effect of adjuvant S-1 therapy $[8,9]$. Our study showed that high TS and low TP expressions were associated with good survival, while no such association was found with the expression of DPD or OPRT. A possible reason for these conflicting results may be the measurement methods used. Many studies have evaluated protein expression using immunohistochemistry or gene expression via RT-PCR at the tumor site, whereas we used microarray and measured normalized gene expression by calculating the ratio between tumor sites and normal tissues. There is no study validating these different methodologies for measurement of S-1 metabolic enzyme levels or assessing the relationship between clinical outcome and their expression levels.

We aimed to identify predictive biomarkers of adjuvant S-1 chemotherapy benefit, in addition to the key enzymes in the S-1 metabolism pathway; therefore, we conducted GEP using cDNA microarray. We found that immune-related genes and GO terms were upregulated and enriched in the favorable outcome group. Several studies have reported that 

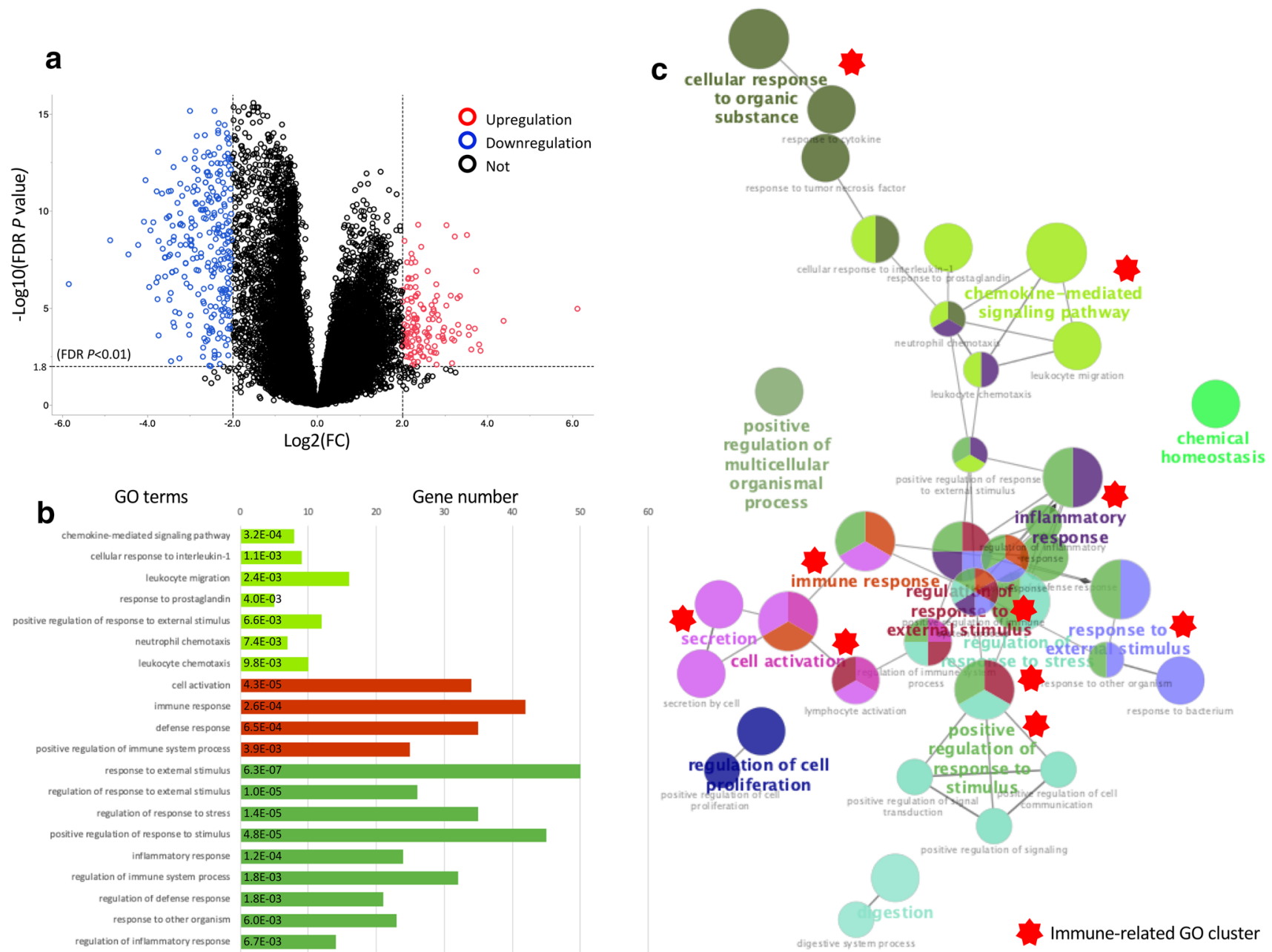

Fig. 2 Identification of DEGs and GO analysis. a Volcano plot of DEGs. The red dots represent upregulated genes and the blue dots represent downregulated genes in cluster A. b Top three significant GO terms. The vertical items show the names of the GO terms. The horizontal axis and length of the graph represent gene numbers. The numbers in the bar chart are adjusted $\mathrm{P}$ values and the colors denote

immune activation is associated with the effect of adjuvant chemotherapy in gastric cancer [18-20]. A recent retrospective study demonstrated that high infiltration of immune cells was associated with a better response to adjuvant chemotherapy in gastric cancer [18]. Most of these studies evaluated immune-cell infiltration using immunohistochemistry staining. Our analysis using microarray data also suggested that immune activation was a predictive biomarker of adjuvant chemotherapy. These results, obtained using different methodologies, consistently indicate the positive impact of immune activation on the benefit of S-1 adjuvant treatment.

Possible mechanisms of antitumor immune response in chemotherapy have been explored. The chemotherapy agent 5 -fluorouracil can partially reduce or temporally inactivate tumor-protective regulatory $\mathrm{T}$ cells and myeloid-derived

the different clusters. c GO network. The GO terms were classified into several functional groups (different node color) based on the Kappa score. Each node represents a GO term, with the size proportional to the significance of the term. The most significant GO terms are labelled with a highlighted color. The edges of the nodes indicate that they share common genes

suppressor cells, which are regulated by various chemokines [21-24]. In the present study, GO analysis revealed the enrichment of the GO term "Chemokine-mediated signaling pathway (GO:0070098)," and PPI network analysis showed that several chemokines were strongly interacting with each other. A previous retrospective study showed that activation of the CXCL13-CXCR5 axis was positively associated with the improved survival outcome in adjuvant chemotherapy comprising of 5-fluorouracil for breast cancer treatment [25]. However, direct associations between the effect of 5-fluorouracil on immune cells and chemokine function have not been fully investigated.

There are several limitations in the current study. First, it was a single-institution retrospective study with a small sample size, possibly leading to selection bias. In 


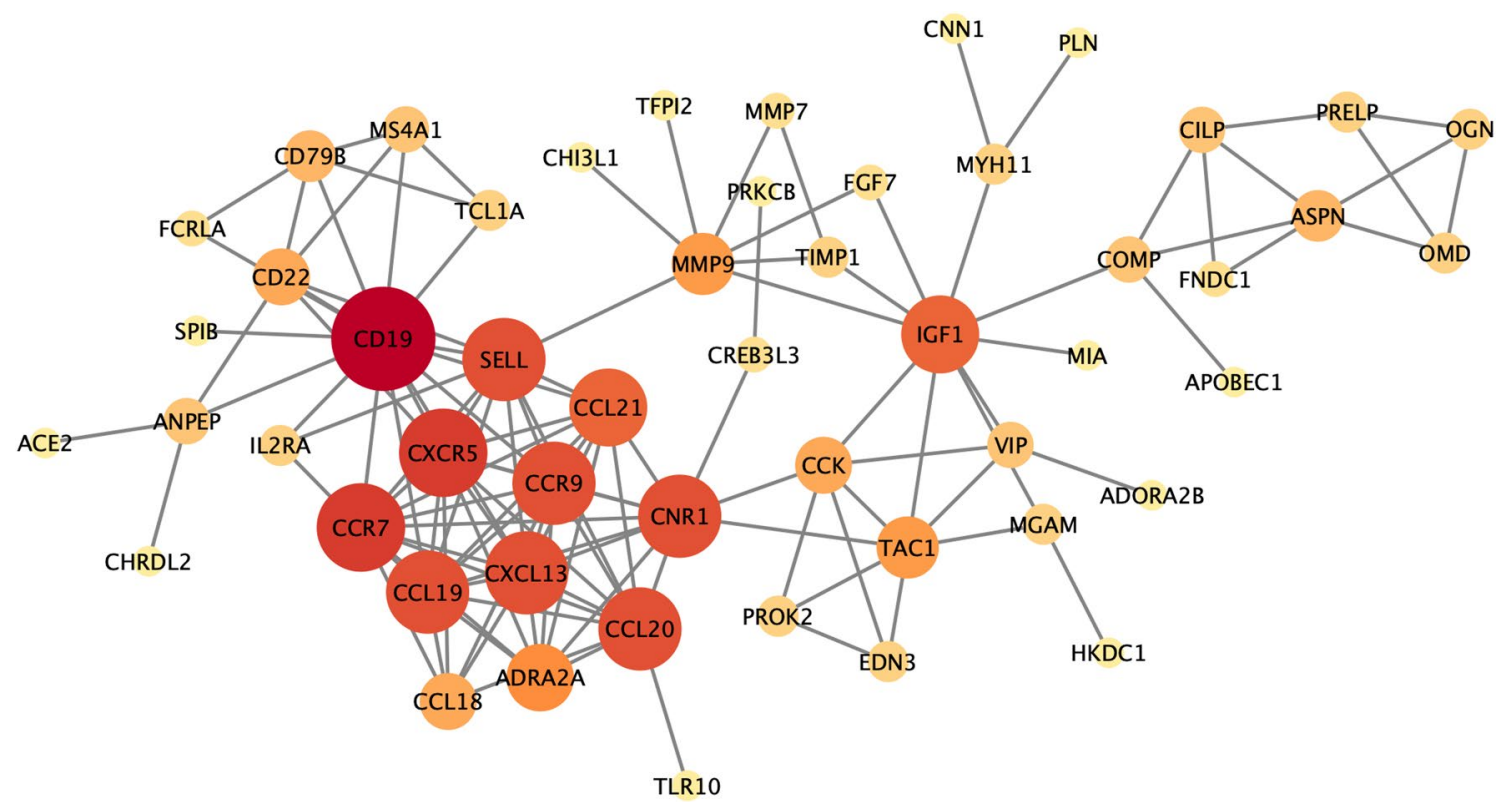

Fig. 3 PPI network analysis. Visualization of the PPI network using the degree method. The red nodes represent the genes most closely related to other genes
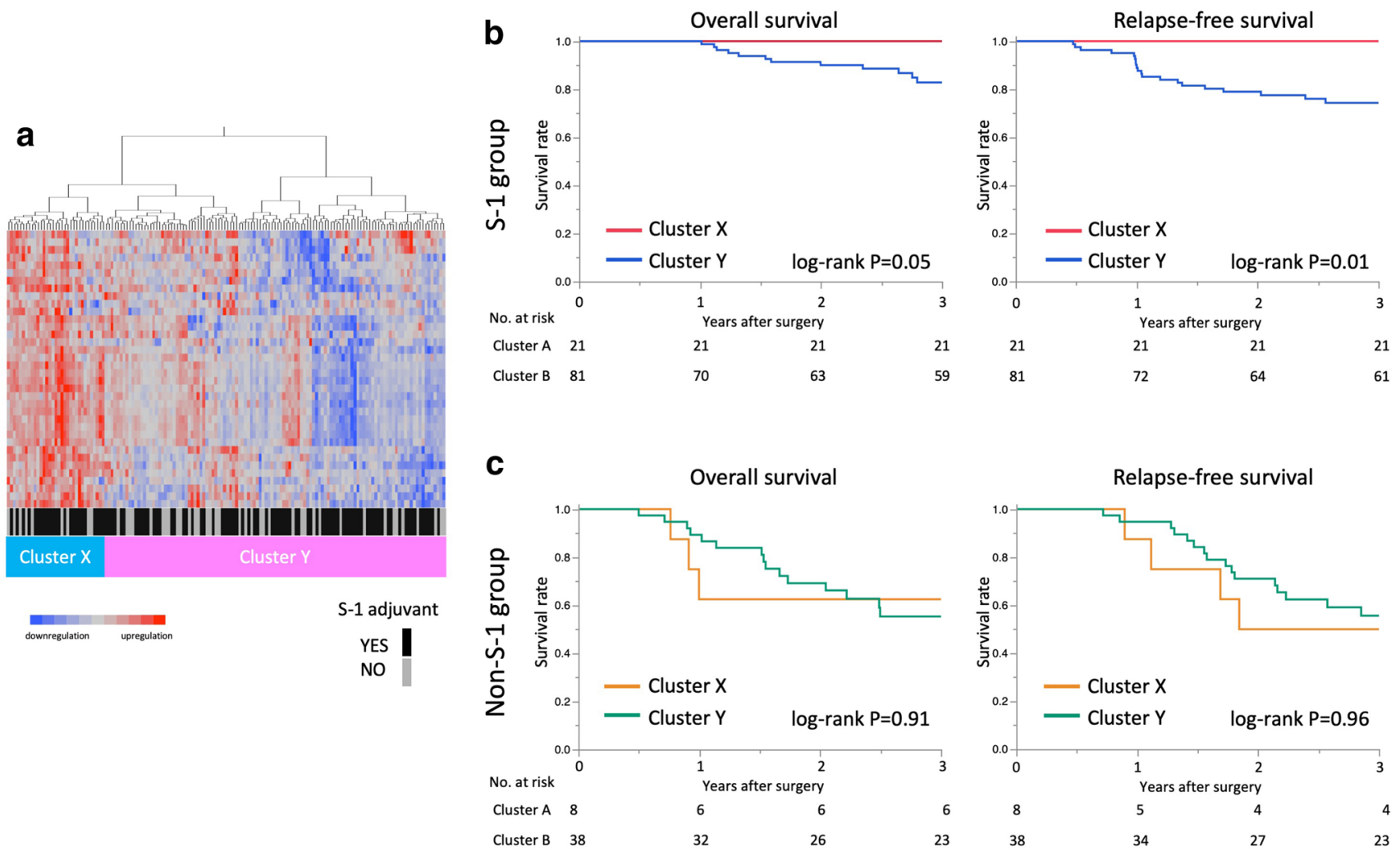

Fig. 4 Impact of upregulation of immune-related genes on survival in the non-S-1 group. a Heatmap based on hierarchical clustering analysis of immune-related genes. The color from blue to red represents the range of gene expression levels from a downregulated state to an upregulated state. The horizontal axis represents patients and the vertical axis represents gene clusters. b OS and RFS curves of clusters $X$ and $\mathrm{Y}$ in the $\mathrm{S}-1$ group. $\mathrm{c}$ OS and RFS curves of clusters $\mathrm{X}$ and $\mathrm{Y}$ in the non-S-1 group 
Table 3 Comparison of patient characteristics between clusters $\mathrm{X}$ and $\mathrm{Y}$ with or without $\mathrm{S}-1$ adjuvant chemotherapy

\begin{tabular}{|c|c|c|c|c|c|c|}
\hline \multirow[t]{2}{*}{ Patient characteristics } & \multicolumn{2}{|l|}{ S-1 group } & \multirow[t]{2}{*}{$P$ value } & \multicolumn{2}{|c|}{ Non-S-1 group } & \multirow[t]{2}{*}{$P$ value } \\
\hline & Cluster X & Cluster Y & & Cluster X & Cluster Y & \\
\hline Total number & 21 & 81 & & 8 & 38 & \\
\hline Age & & & 0.29 & & & 0.07 \\
\hline Median (range) & $67(23-77)$ & $69(31-79)$ & & $83(79-88)$ & $80(48-88)$ & \\
\hline Sex & & & 0.97 & & & 0.16 \\
\hline Male & $16(76)$ & $62(77)$ & & $4(50)$ & $26(68)$ & \\
\hline Female & $5(24)$ & $19(23)$ & & $4(50)$ & $12(32)$ & \\
\hline Tumor location & & & 0.08 & & & 0.43 \\
\hline Upper & $7(33)$ & $25(31)$ & & $1(12)$ & $11(29)$ & \\
\hline Middle & $3(14)$ & $30(37)$ & & $3(38)$ & $16(42)$ & \\
\hline Lower & $11(53)$ & $26(32)$ & & $4(50)$ & $11(29)$ & \\
\hline Histological type & & & 0.46 & & & 0.52 \\
\hline Differentiated & $12(57)$ & $39(48)$ & & $5(62)$ & $19(50)$ & \\
\hline Undifferentiated & $9(43)$ & $42(52)$ & & $3(38)$ & $19(50)$ & \\
\hline $\mathrm{T}$ & & & 0.63 & & & 0.26 \\
\hline $\mathrm{T} 1$ & $0(0)$ & $1(1)$ & & $1(12)$ & $1(3)$ & \\
\hline $\mathrm{T} 2$ & $2(10)$ & $14(17)$ & & $3(38)$ & $7(18)$ & \\
\hline $\mathrm{T} 3$ & $9(43)$ & $26(32)$ & & $2(25)$ & $7(18)$ & \\
\hline $\mathrm{T} 4$ & $10(47)$ & $40(50)$ & & $2(25)$ & $23(61)$ & \\
\hline $\mathrm{N}$ & & & 0.35 & & & 0.28 \\
\hline No & $2(10)$ & $4(5)$ & & $0(0)$ & $6(16)$ & \\
\hline N1 & $5(24)$ & $26(32)$ & & $4(50)$ & $11(29)$ & \\
\hline $\mathrm{N} 2$ & $10(47)$ & $25(31)$ & & $3(38)$ & $11(29)$ & \\
\hline N3 & $4(19)$ & $26(32)$ & & $1(12)$ & $10(26)$ & \\
\hline Stage & & & 0.99 & & & 0.84 \\
\hline II & $8(38)$ & $31(38)$ & & $4(50)$ & $17(46)$ & \\
\hline III & $13(62)$ & $50(62)$ & & $4(50)$ & $21(54)$ & \\
\hline Type of gastrectomy & & & 0.39 & & & 0.19 \\
\hline Proximal & $1(5)$ & $2(2)$ & & $0(0)$ & $0(0)$ & \\
\hline Distal & $14(67)$ & $43(53)$ & & $7(88)$ & $25(66)$ & \\
\hline Total & $6(28)$ & $36(45)$ & & $1(12)$ & $13(34)$ & \\
\hline
\end{tabular}

particular, patients not receiving adjuvant S-1 were elderly and had multiple comorbidities. Although the upregulation of immune-related genes was not associated with survival in the patients not receiving adjuvant $\mathrm{S}-1$, the possible selection bias may have influenced our findings regarding tumor immune response [26, 27]. However, it is evident from our data that there were no significant differences in the agebased immune-related gene expressions between the S-1 and the non-S-1 groups (Supplementary Fig. 2a, b). Second, we did not analyze microsatellite instability (MSI) status or EBV-infection in all patients. Although it has been reported that MSI-high gastric cancers or EBV-associated gastric cancers are lymphocyte-rich tumors and show favorable survival outcome [28, 29], the distribution of these status in each clusters was unknown. We evaluate EBV-infection by EBV-encoded small RNA in situ hybridization (EBERISH) in case of gastric carcinoma with lymphoid stroma (GCLS). Although we did not evaluate all samples, there was no significant difference in EBER-ISH positive gastric cancer between cluster A and B (1/28 in cluster A vs 5/74 in cluster $\mathrm{B}, P=0.52)$, and cluster $\mathrm{X}$ and $\mathrm{Y}(0 / 29$ in cluster $\mathrm{X}$ vs $7 / 119$ in cluster $\mathrm{Y}, P=0.08$ ), respectively. Third, the noncancerous tissue was defined based on only macroscopic findings. We did not evaluate histological features of the noncancerous tissues, including normal mucosa, atrophic mucosa, or intestinal metaplasia. These epithelial changes could correlate with the alteration of gene expression due to DNA methylation. Therefore, the definition of noncancerous tissue in gastric cancer should be carefully evaluated by histological findings or measurement of DNA methylation level in future analysis. Fourth, we could not validate the prognostic impact of the upregulation of immune-related genes in the different cohorts. Therefore, a multi-institution prospective study is needed to validate our results.

In conclusion, we found that upregulation of immunerelated genes was associated with a favorable survival 
outcome in patients receiving adjuvant S-1 chemotherapy. Our findings suggest that assessment of immune response, a likely predictive biomarker, serves as a method to identify patients who would benefit from S-1 adjuvant chemotherapy.

Acknowledgements The authors would like to thank Enago (https:// www.enago.jp) for the English language review.

Funding This work was supported by the National Cancer Center Research and Development Funds (29-A-3) and the Japan Society for the Promotion of Science, KAKENHI Grant No. 16K10523.

\section{Compliance with ethical standards}

Conflict of interest The authors declare that they have no conflict of interest.

Ethical standards All procedures followed were in accordance with the ethical standards of the responsible committee on human experimentation (institutional and national) and with the Helsinki Declaration of 1964 and later versions. Informed consent was included in the study, or its equivalent, was obtained from all patients.

\section{References}

1. Bray F, Ferlay J, Soerjomataram I, Siegel RL, Torre LA, Jemal A. Global cancer statistics 2018: GLOBOCAN estimates of incidence and mortality worldwide for 36 cancers in 185 countries. CA Cancer J Clin. 2018;68:394-424.

2. Japanese Gastric Cancer A. Japanese gastric cancer treatment guidelines 2014 (ver. 4). Gastric Cancer. 2017;20:1-19.

3. Sakuramoto S, Sasako M, Yamaguchi T, Kinoshita T, Fujii M, Nashimoto A, et al. Adjuvant chemotherapy for gastric cancer with S-1, an oral fluoropyrimidine. N Engl J Med. 2007;357:1810-20.

4. Sasako M, Sakuramoto S, Katai H, Kinoshita T, Furukawa H, Yamaguchi T, et al. Five-year outcomes of a randomized phase III trial comparing adjuvant chemotherapy with $\mathrm{S}-1$ versus surgery alone in stage II or III gastric cancer. J Clin Oncol. 2011;29:4387-93.

5. Sasako M, Terashima M, Ichikawa W, Ochiai A, Kitada K, Kurahashi I, et al. Impact of the expression of thymidylate synthase and dihydropyrimidine dehydrogenase genes on survival in stage II/III gastric cancer. Gastr Cancer. 2015;18:538-48.

6. Ichikawa W, Takahashi T, Suto K, Yamashita T, Nihei Z, Shirota $\mathrm{Y}$, et al. Thymidylate synthase predictive power is overcome by irinotecan combination therapy with $\mathrm{S}-1$ for gastric cancer. $\mathrm{Br}$ J Cancer. 2004;91:1245-50.

7. Shimizu T, Yamada Y, Yasui H, Shirao K, Fukuoka M. Clinical application of immunoreactivity of dihydropyrimidine dehydrogenase (DPD) in gastric scirrhous carcinoma treated with S-1, a new DPD inhibitory fluoropyrimidine. Anticancer Res. 2005;25:2997-3001.

8. Miyamoto S, Boku N, Ohtsu A, Yoshida S, Ochiai A, Okabe H, et al. Clinical implications of immunoreactivity of thymidylate synthase and dihydropyrimidine dehydrogenase in gastric cancer treated with oral fluoropyrimidine (S-1). Study Group of S-1 for Gastric Cancer. Int J Oncol. 2000;17:653-8.

9. Ichikawa W. Prediction of clinical outcome of fluoropyrimidinebased chemotherapy for gastric cancer patients, in terms of the 5-fluorouracil metabolic pathway. Gastr Cancer. 2006;9:145-55.
10. Wang D, Yu X, Wang X. High/positive expression of 5-fluorouracil metabolic enzymes predicts better response to $\mathrm{S}-1$ in patients with gastric cancer: a meta-analysis. Int J Biol Mark. 2016;31:e101-109.

11. Cancer Genome Atlas Research N. Comprehensive molecular characterization of gastric adenocarcinoma. Nature. 2014;513:202-9.

12. Yamaguchi K, Urakami K, Ohshima K, Mochizuki T, Akiyama Y, Uesaka K, et al. Implementation of individualized medicine for cancer patients by multiomics-based analyses-the Project HOPE. Biomed Res. 2014;35:407-12.

13. Schroeder A, Mueller O, Stocker S, Salowsky R, Leiber M, Gassmann M, et al. The RIN: an RNA integrity number for assigning integrity values to RNA measurements. BMC Mol Biol. 2006; 7:3.

14. Hatakeyama K, Ohshima K, Nagashima T, Ohnami S, Ohnami $\mathrm{S}$, Serizawa M, et al. Molecular profiling and sequential somatic mutation shift in hypermutator tumours harbouring POLE mutations. Sci Rep. 2018;8:8700.

15. Ohshima K, Hatakeyama K, Nagashima T, Watanabe Y, Kanto $\mathrm{K}$, Doi $\mathrm{Y}$, et al. Integrated analysis of gene expression and copy number identified potential cancer driver genes with amplification-dependent overexpression in 1,454 solid tumors. Sci Rep. 2017;7:641.

16. Brazma A. Minimum Information About a Microarray Experiment (MIAME)—successes, failures, challenges. Sci World J. 2009;9:420-3.

17. Bindea G, Mlecnik B, Hackl H, Charoentong P, Tosolini M, Kirilovsky A, et al. ClueGO: a Cytoscape plug-in to decipher functionally grouped gene ontology and pathway annotation networks. Bioinformatics. 2009;25:1091-3.

18. Jiang Y, Zhang Q, Hu Y, Li T, Yu J, Zhao L, et al. ImmunoScore signature: a prognostic and predictive tool in gastric cancer. Ann Surg. 2018;267:504-13.

19. Wang B, Xu D, Yu X, Ding T, Rao H, Zhan Y, et al. Association of intra-tumoral infiltrating macrophages and regulatory $\mathrm{T}$ cells is an independent prognostic factor in gastric cancer after radical resection. Ann Surg Oncol. 2011;18:2585-93.

20. Wang JT, Li H, Zhang H, Chen YF, Cao YF, Li RC, et al. Intratumoral IL17-producing cells infiltration correlate with antitumor immune contexture and improved response to adjuvant chemotherapy in gastric cancer. Ann Oncol. 2019;30:266-73.

21. Galluzzi L, Buque A, Kepp O, Zitvogel L, Kroemer G. Immunological effects of conventional chemotherapy and targeted anticancer agents. Cancer Cell. 2015;28:690-714.

22. Zitvogel L, Apetoh L, Ghiringhelli F, Andre F, Tesniere A, Kroemer $\mathrm{G}$. The anticancer immune response: indispensable for therapeutic success? J Clin Invest. 2008;118:1991-2001.

23. Coffelt SB, de Visser KE. Immune-mediated mechanisms influencing the efficacy of anticancer therapies. Trends Immunol. 2015;36:198-21616.

24. Vincent J, Mignot G, Chalmin F, Ladoire S, Bruchard M, Chevriaux A, et al. 5-Fluorouracil selectively kills tumorassociated myeloid-derived suppressor cells resulting in enhanced $\mathrm{T}$ cell-dependent antitumor immunity. Cancer Res. 2010;70:3052-61.

25. Razis E, Kalogeras KT, Kotoula V, Eleftheraki AG, Nikitas N, Kronenwett R, et al. Improved outcome of high-risk early HER2 positive breast cancer with high CXCL13-CXCR5 messenger RNA expression. Clin Breast Cancer. 2012;12:183-93.

26. Derhovanessian E, Solana R, Larbi A, Pawelec G. Immunity, ageing and cancer. Immun Age. 2008;5:11.

27. Aunan JR, Watson MM, Hagland HR, Soreide K. Molecular and biological hallmarks of ageing. Br J Surg. 2016;103:e29-46.

28. Polom K, Marano L, Marrelli D, De Luca R, Roviello G, Savelli $\mathrm{V}$, et al. Meta-analysis of microsatellite instability in relation to 
clinicopathological characteristics and overall survival in gastric cancer. Br J Surg. 2018;105:159-67.

29. Sohn BH, Hwang JE, Jang HJ, Lee HS, Oh SC, Shim JJ, et al. Clinical significance of four molecular subtypes of gastric cancer identified by The Cancer Genome Atlas Project. Clin Cancer Res. 2017;23:4441-9.
Publisher's Note Springer Nature remains neutral with regard to jurisdictional claims in published maps and institutional affiliations. 